\title{
Ethnomedicinal Analysis of Toxic Plants from Five Ethnic Groups in China
}

\author{
Huyin Huai, Qinqin Dong and Aizhong Liu
}

\section{Research}

\begin{abstract}
Toxic plants are important elements of ethnomedicine. Ethnomedicinal knowledge on toxic plants recorded from five indigenous people, Dai, Lahu, Miao, Tujia, and Wa in south and southwestern China, was summarized and analyzed based on available literature. A formula has been developed to evaluate toxic plants in the ethnomedicine of different ethnic groups using the Average Use Values (AUVs) of them. In total, 118 toxic plants often used as ethnomedicines were found from the five ethnic groups. These toxic plants were mainly distributed in 21 families, i.e., $75.4 \%$ of species and $68.1 \%$ of genera were concentrated in the 21 families. Araceae, Asteraceae, Euphorbiaceae, and Fabaceae are 4 important families which contain rich toxic plants. Eleven toxic plants were thought to be deadly toxic. These toxic plants were often used medicinally to treat injuries from falls, broken bones, and skin problems. Most toxic plants were medicinally used for multiple purposes in the five ethnic groups. The medicinal role of toxic plants was ascertained by comparing the AUVs or UVs in these ethnomedicines. The culture and the resources available were two main factors affecting ethnic healers selecting and using toxic plants.
\end{abstract}

\section{Introduction}

Poisonous plants are considered as detrimental to or adversely affecting human bodies and animals (Eddouks et al. 2002, Huai \& Xu 2000, Levetin \& McMahon 2008). However, toxins from plants are closely related to health aspects of humans and animals (Habermehl 2004, Levetin \& McMahon 2008, Rates 2001) with "toxic" constituents from them often applied as effective treatments of some refractory symptoms of human disease (Harvey et al. 1998). The taxine alkaloid, for example, is a poisonous compound from yews (Taxus spp.) but has been found to have positive pharmacological functions in the treatment of cancer (Wang et al. 2005, Wilson et al. 2001). Toxic plants are very useful sources of compounds identified through screens to develop new drugs (Heinrich 2000, Rates 2001, Vetter 2000).

Indigenous knowledge is a very important and inexhaustible "information bank" for toxin research (Huai \& Xu 2000). Plants that generally cause human beings or animals to have symptoms such as vomiting, stomach cramps, or difficulty breathing, are considered as "toxic plants" in traditional communities. Indigenous people all over the world are usually good at using toxic plants for different aims, such as hunting, wars, and treating diseases (Al-Qura'n 2005, Levetin \& McMahon 2008, Neuwinger 2004). Some "toxic" plants are also nutritious (Bhattarai et al. 2009, Stinson 1992).

In traditional Chinese medicine (including ethnomedicine and folk medicine), "toxic" plants are commonly used to treat different health problems. This phenomenon is similar in other folk medicinal systems elsewhere

\section{Correspondence}

Huyin Huai, College of Bioscience and Biotechnology, Yangzhou University, Yangzhou 225009, P.R. CHINA.

Qinqin Dong, College of Bioscience and Biotechnology, Yangzhou University, Yangzhou 225009, P.R. CHINA.

Aizhong Liu, Xishuangbanna tropical Botanical Garden, Chinese Academy of Sciences, Kunming 650223, P.R. CHINA.

liuaizhong@xtbg.ac.cn

Ethnobotany Research \& Applications 8:169-179 (2010)

Published: June 30, 2010 
in the world (Al-Qura'n 2005, Arias 2000). Much Chinese ethnobotanical knowledge on toxic plants has been recorded in recent years (Guo et al. 1990, Guo et al. 1991, Qiu et al. 2006, Zhang 1996, Zhu et al. 2006). Previous studies on toxic plants focused on their chemical compositions and pharmacological effects (e.g., Farnsworth 1966, Rios \& Waterman 1998, Vetter 2000). However, little is known about the relationships between the uses of toxic plants and their taxonomic and cultural contexts in different ethnic groups. Different ethnic groups may have their own knowledge and experiences of using plant resources even if they inhabit the same environments. In this paper, we focused on the toxic plants as reported in ethnomedicine sources on five ethic groups in south and southwest China and tried to answer the following questions: What health problems do ethnic healers usually use toxic plants to treat? Is there any difference in use of the same toxic plant by healers with different cultural backgrounds? Are there any correlations between toxic plants and use? All of the answers to these questions are important guidelines for the exploration of toxic bioresources.

\section{Methods and materials}

The Dai, Lahu, Miao, Tujia, and Wa ethnic groups live in mountainous areas of south and southwest China. Ethnobotanical investigations on their traditional medicine have been conducted and published in recent years (e.g., Dai (Lin et al. 2003b), Lahu (Zhang 1996), Lahu (Simao Institute of Ethnomedicine 1987), Miao (Qiu et al. 2006), Tujia (Zhu et al. 2006), and Wa (Guo et al. 1990, Guo et al. 1991). Toxic plants are commonly used in these ethnomedicinal systems. As our basic method, we have conducted a metadata analysis through harvesting of results from the above published materials. The information includes species name, family name, part used, use, preparation, toxicity, and special use.

Thus far, there are not standardized quantitative methods for evaluating the toxicity of a plant in ethnomedicine. Indigenous people often evaluate the toxicity of a plant based on their experiences. Here, we classified the toxicity of poisonous plants into three groups, i.e., low, medium, and high, based on the records of toxicity reported in the published sources. A "high" level means that this plant contains deadly toxins and leads a person or animal to quick death, even if only a small amount is taken. "Low" means that the plant can cause somebody poisoning when a great amount is used or it is used incorrectly. A "medium" plant is intermediary between "low" and "high."
The formula suggested by Phillips and Gentry (1993a \& b) was used to evaluate the medicinal use value (UVS) of each species $s$ :

$U V S=\frac{\sum_{i=1}^{n} U_{i s}}{n_{s}}$

where $U_{i s}$ was the number of medicinal uses of species $s$ mentioned by informant $i$, and $n s$ was the number of informants who mentioned species $s$. Here, since this is a meta-data analysis of published texts (that could represent few too many interviews but are published as a single summary), $i$ and $n s$ have been treated as one for each ethnomedicinal system, while the number of ethnomedicines which was recorded for species $s$ fluxuates. Based on this formula, when only one use of species $s$ was recorded in only one ethnomedicine, its UVS was 1; if one use of another species was recorded by 4 ethnomedicinal systems respectively, its UVs was 1 as well. Because we were not tracking differences in the number of users but only in the uses per species with UVs it was very difficult to identify differences. In order to avoid this problem we developed a concept of average UVs (AUVs). AUVs can be calculated by the following formula:

$A U V_{s}=U V_{s} \times P_{s}$

where UVs was calculated based on the formula suggested by Phillips and Gentry (1993a \& b). \% $P_{s}$ was the percentage of the ethnomedicinal systems examined that mentioned species $s$.

The relationships among the five ethnomedicinal systems and their use of toxic plants were analyzed by SPSS 16.0 (SPSS 2008) applying a Pearson's product-moment correlation.

\section{Results and discussion}

\section{The diversity of toxic plants in the five ethnomedicine}

In total, 118 species of ethnomedicinally toxic plants from the five ethnic groups were inventoried (Table 1), covering 51 families and 92 genera. The average ratio of number of species to family was 2.31 . Twenty families (39.2\%) contained at least two species of toxic plants (Table 2).

Table 1. Inventory of toxic medicinal plants recorded in Dai, Lahu, Miao, Tujia, and Wa ethnic groups in south and southwest China.

\begin{tabular}{|c|c|c|c|}
\hline Plant Family/Species & Part Used & Toxicity & Reference \\
\hline \multicolumn{4}{|l|}{ Apocynaceae } \\
\hline Alstonia scholaris (L.) R.Br. & Bark, leaf & low & Guo et al. 1990 \\
\hline Kopsia officinalis Tsiang \& P.T. Li & Fruit & low & Lin et al. 2003 \\
\hline
\end{tabular}


Huai et al. - Ethnomedicinal Analysis of Toxic Plants from Five Ethnic Groups in China

\begin{tabular}{|c|c|c|c|}
\hline Plant Family/Species & Part Used & Toxicity & Reference \\
\hline Nerium oleander L. & Leaf, bark & high & Zhu et al. 2006 \\
\hline Periploca forrestii Schltr. & Root, whole plant & low & Qiu et al. 2006 \\
\hline Rauvolfia verticillata (Lour.) Baill. & Root & low & Lin et al. 2003 \\
\hline \multicolumn{4}{|l|}{ Araceae } \\
\hline Alocasia macrorrhizos (L.) G. Don & Stem & medium & Guo et al. 1991 \\
\hline Amorphophallus rivieri Durieu ex Carrière & Tuber & medium & Zhu et al. 2006 \\
\hline Amydrium sinense (Engl.) H. Li & Whole plant & medium & Zhu et al. 2006 \\
\hline Arisaema consanguineum Schott & Corm & high & Zhu et al. 2006 \\
\hline Arisaema erubescens (Wall.) Schott & Tuber & medium & Guo et al. 1991, Qiu et al. 2006 \\
\hline Arisaema fargesi Buchet & Tuber & low & Zhu et al. 2006 \\
\hline Arisaema rhizomatum C.E.C. Fisch. & Tuber & medium & Zhu et al. 2006 \\
\hline Colocasia esculenta (L.) Schott & Rhizome & low & Zhang, 1996 \\
\hline Epipremnum pinnatum (L.) Engl. & Whole plant & low & Lin et al. 2003 \\
\hline Pinellia ternata (Thunb.) Breitenb. & Tuber & medium & Zhu et al. 2006, Qiu et al. 2006 \\
\hline Remusatia vivipara (Roxb.) Schott & Whole plant, tuber & high & Guo et al. 1991, Zhang, 1996 \\
\hline Typhonium roxburghii Schott & Whole plant, tuber & medium & Guo et al. 1991 \\
\hline Typhonium trilobatum (L.) Schott & Tuber, leaf & medium & Lin et al. 2003 \\
\hline \multicolumn{4}{|l|}{ Aristolochiaceae } \\
\hline Aristolochia debilis Siebold \& Zucc. & root & low & Qiu et al. 2006 \\
\hline $\begin{array}{l}\text { Aristolochia griffithii Hook. f. } \\
\text { \& Thomson ex Duch. }\end{array}$ & root & low & Anonymous 1987 \\
\hline Aristolochia tubiflora Dunn & Root, whole plant & low & Qiu et al. 2006 \\
\hline Asarum sieboldii Miq. & Root, whole plant & low & Zhu et al. 2006 \\
\hline Asarum wulingense C.F. Liang & Whole plant & low & Qiu et al. 2006 \\
\hline Saruma henryi Oliv. & Root, rhizome & low & Zhu et al. 2006 \\
\hline \multicolumn{4}{|l|}{ Asparagaceae } \\
\hline Hosta plantaginea (Lam.) Asch. & Flower & medium & Zhu et al. 2006 \\
\hline \multicolumn{4}{|l|}{ Asteraceae } \\
\hline Achillea wilsoniana Heimerl & Whole plant & medium & Qiu et al. 2006 \\
\hline Carpesium abrotanoides L. & Whole plant & low & Zhu et al. 2006 \\
\hline Eupatorium chinense L. & Whole plant & medium & Qiu et al. 2006 \\
\hline Gynura procumbens (Lour.) Merr. & Whole plant & low & Lin et al. 2003 \\
\hline Siegesbeckia pubescens (Makino) Makino & Whole plant & low & Qiu et al. 2006 \\
\hline Solidago decurrens Lour. & Whole plant & low & Zhu et al. 2006 \\
\hline Spilanthes callimorpha A.H. Moore & Whole plant & low & Guo et al. 1990, Lin et al. 2003 \\
\hline Spilanthes paniculata Wall. ex DC. & Whole plant & medium & Guo et al. 1990 \\
\hline \multicolumn{4}{|l|}{ Balsaminaceae } \\
\hline Impatiens uliginosa Franch. & Whole plant & low & Qiu et al. 2006 \\
\hline \multicolumn{4}{|l|}{ Berberidaceae } \\
\hline $\begin{array}{l}\text { Dysosma difformis (Hemsl. \& } \\
\text { E.H. Wilson) T.H. Wang }\end{array}$ & \multirow[t]{2}{*}{ Root, rhizome } & \multirow[t]{2}{*}{ medium } & \multirow[t]{2}{*}{ Zhu et al. 2006, Qiu et al. 2006} \\
\hline Dysosma majorensis (Gagnep.) T.S. Ying & & & \\
\hline
\end{tabular}




\begin{tabular}{|c|c|c|c|}
\hline Plant Family/Species & Part Used & Toxicity & Reference \\
\hline $\begin{array}{l}\text { Dysosma veitchii (Hemsl. \& } \\
\text { E.H. Wilson) Fu ex Ying }\end{array}$ & \multirow[t]{2}{*}{ Root, rhizome } & \multirow[t]{2}{*}{ medium } & \multirow[t]{2}{*}{ Zhu et al. 2006, Quu et al. 2006} \\
\hline $\begin{array}{l}\text { Dysosma versipellis (Hance) } \\
\text { M. Cheng ex T.S. Ying }\end{array}$ & & & \\
\hline Nandina domestica Thunb. & Fruit, root & medium & Qiu et al. 2006 \\
\hline \multicolumn{4}{|l|}{ Cactaceae } \\
\hline Opuntia stricta (Haw.) Haw. & Root, stem & low & Qiu et al. 2006 \\
\hline \multicolumn{4}{|l|}{ Campanulaceae } \\
\hline Lobelia clavata E. Wimm. & Root, leaf & high & Guo et al. 1991 \\
\hline $\begin{array}{l}\text { Lobelia colorata subsp. taliensis } \\
\text { (Diels) T.J. Zhang \& D.Y. Hong }\end{array}$ & Root & low & Guo et al. 1991 \\
\hline \multicolumn{4}{|l|}{ Caryophyllaceae } \\
\hline $\begin{array}{l}\begin{array}{l}\text { Psammosilene tunicoides } \\
\text { W.C. Wu \& C.Y. Wu }\end{array} \\
\end{array}$ & Root & low & Qiu et al. 2006 \\
\hline \multicolumn{4}{|l|}{ Chloranthaceae } \\
\hline Chloranthus erectus (Buch.-Ham.) Verdc. & Whole plant & low & Guo et al. 1990 \\
\hline Chloranthus henryi HemsI. & Root & low & Zhu et al. 2006 \\
\hline $\begin{array}{l}\text { Chloranthus holostegius (Hand.- } \\
\text { Mazz.) S.J. Pei \& Shan }\end{array}$ & Root, leaf & low & Guo et al. 1991 \\
\hline \multicolumn{4}{|l|}{ Colchiaceae } \\
\hline Gloriosa superba L. & Rhizome & high & Lin et al. 2003 \\
\hline \multicolumn{4}{|l|}{ Combretaceae } \\
\hline Quisqualis indica L. & Fruit & low & Guo et al. 1991 \\
\hline \multicolumn{4}{|l|}{ Convolvulaceae } \\
\hline Pharbitis purpurea (L.) Voigt & Seed & medium & Qiu et al. 2006 \\
\hline \multicolumn{4}{|l|}{ Coriariaceae } \\
\hline Coriaria sinica Maxim. & Root, leaf & medium & Zhu et al. 2006, Qiu et al. 2006 \\
\hline \multicolumn{4}{|l|}{ Cornaceae } \\
\hline Alangium chinense (Lour.) Harms & Root, root bark & medium & Zhu et al. 2006, Qu et al. 2006 \\
\hline \multicolumn{4}{|l|}{ Costaceae } \\
\hline Costus speciosus (J. König) Sm. & Rhizome & low & Lin et al. 2003 \\
\hline \multicolumn{4}{|l|}{ Cucurbitaceae } \\
\hline Trichosanthes cucumeroides (Ser.) Maxim. & Fruit & medium & Zhu et al. 2006 \\
\hline \multicolumn{4}{|l|}{ Dioscoreaceae } \\
\hline Dioscorea bulbifera L. & Tuber, leaf & low & Guo et al. 1991, Zhu et al. 2006 \\
\hline Dioscorea cirrhosa Lour. & Tuber & low & Qiu et al. 2006 \\
\hline Dioscorea hispida Dennst. & Tuber & medium & $\begin{array}{l}\text { Anonymous } 1987, \\
\text { Guo et al. } 1990\end{array}$ \\
\hline \multicolumn{4}{|l|}{ Dipterocarpaceae } \\
\hline Dipterocarpus turbinatus Gaertn. & Leaf & medium & Lin et al. 2003 \\
\hline \multicolumn{4}{|l|}{ Ericaceae } \\
\hline $\begin{array}{l}\text { Gaultheria leucocarpa BI. var. } \\
\text { crenulata (Kurz) T.Z. Hsu }\end{array}$ & Whole plant, root & low & Qiu et al. 2006 \\
\hline
\end{tabular}


Huai et al. - Ethnomedicinal Analysis of Toxic Plants from Five Ethnic Groups in China

\begin{tabular}{|c|c|c|c|}
\hline Plant Family/Species & Part Used & Toxicity & Reference \\
\hline \multicolumn{4}{|l|}{ Euphorbiaceae } \\
\hline Croton caudatus Geiseler & Whole plant & low & Lin et al. 2003 \\
\hline Croton tiglium L. & Seed, leaf & high & Guo et al. 1991, Lin et al. 2003 \\
\hline Euphorbia antiquorum L. & Stem, leaf & medium & Guo et al. 1991, Lin et al. 2003 \\
\hline Euphorbia lathyris L. & Whole plant, seed & medium & Zhu et al. 2006, Qiu et al. 2006 \\
\hline Glochidion puberum (L.) Hutch. & Root, fruit & low & Qiu et al. 2006 \\
\hline Jatropha curcas L. & Bark, leaf & medium & Guo et al. 1991 \\
\hline Ricinus communis $\mathrm{L}$. & Leaf, root, fruit, seed & low & $\begin{array}{l}\text { Guo et al. 1990, Lin et al. 2003, } \\
\text { Quu et al. 2006, Zhu et al. } 2006\end{array}$ \\
\hline \multicolumn{4}{|l|}{ Fabaceae } \\
\hline Adenanthera microsperma Teijsm. \& Binn. & Seed, root, bark, leaf & low & Lin et al. 2003 \\
\hline Caesalpinia minax Hance & Seed & low & Lin et al. 2003 \\
\hline Erythrina arborescens Roxb. & Bark & low & Guo et al. 1990 \\
\hline Millettia pachycarpa Benth. & Root, leaf, seed & high & Guo et al. 1991, Zhang 1996 \\
\hline Ormosia hosiei Hemsl. \& E.H. Wilson & Seed & low & Qiu et al. 2006 \\
\hline $\begin{array}{l}\text { Ormosia nuda (F.C. How) } \\
\text { R.H. Chang \& Q.W. Yao }\end{array}$ & Fruit & low & Qiu et al. 2006 \\
\hline Pachyrhizus erosus (L.) Urb. & Seed, root tuber & medium & Qiu et al. 2006, Lin et al. 2003 \\
\hline \multicolumn{4}{|l|}{ Gelsemiaceae } \\
\hline $\begin{array}{l}\text { Gelsemium elegans (Gardner } \\
\text { \& Champ.) Benth. }\end{array}$ & Whole plant, root & high & Guo et al. 1991, Zhang, 1996 \\
\hline \multicolumn{4}{|l|}{ Ginkgoaceae } \\
\hline Ginkgo biloba L. & Leaf, seed & medium & Zhu et al. 2006 \\
\hline \multicolumn{4}{|l|}{ Hypoxidaceae } \\
\hline Curculigo orchioides Gaertn. & Rhizome & medium & Guo et al. 1991, Zhu et al. 2006 \\
\hline \multicolumn{4}{|l|}{ Iridaceae } \\
\hline $\begin{array}{l}\text { Iris tectorum Maxim. } \\
\end{array}$ & Rhizome & low & Qiu et al. 2006 \\
\hline \multicolumn{4}{|l|}{ Lauraceae } \\
\hline Cassytha filiformis L. & Whole plant & low & Guo et al. 1990, Lin et al. 2003 \\
\hline Litsea glutinosa (Lour.) C.B. Rob. & Root bark, bark, leaf & medium & Lin et al. 2003 \\
\hline \multicolumn{4}{|l|}{ Liliaceae } \\
\hline Rohdea japonica (Thunb.) Roth & Root, rhizome & medium & Zhu et al. 2006, Qiu et al. 2006 \\
\hline Sansevieria trifasciata Prain & Leaf & medium & Lin et al. 2003 \\
\hline \multicolumn{4}{|l|}{ Lycopodiaceae } \\
\hline Huperzia serrata (Thunb.) Trevis. & Whole plant & low & Zhu et al. 2006 \\
\hline \multicolumn{4}{|l|}{ Melanthiaceae } \\
\hline Paris polyphylla Sm. & Rhizome & low & $\begin{array}{l}\text { Guo et al. 1990, Lin et al. } \\
\text { 2003, Qiu et al. } 2006\end{array}$ \\
\hline Veratrum mengtzeanum Loes. & Root & medium & Zhang, 1996 \\
\hline \multicolumn{4}{|l|}{ Meliaceae } \\
\hline Melia azedarach L. & Fruit, bark & medium & Zhu et al. 2006, Qu et al. 2006 \\
\hline \multicolumn{4}{|l|}{ Menispermaceae } \\
\hline Stephania cephalantha Hayata & Root tuber & low & Qiu et al. 2006 \\
\hline
\end{tabular}




\begin{tabular}{|c|c|c|c|}
\hline Plant Family/Species & Part Used & Toxicity & Reference \\
\hline Stephania epigaea H.S. Lo & Root tuber & low & Lin et al. 2003 \\
\hline \multicolumn{4}{|l|}{ Moraceae } \\
\hline Antiaris toxicaria Lesch. & Bark, leaf & high & Lin et al. 2003 \\
\hline \multicolumn{4}{|l|}{ Orchidaceae } \\
\hline Cremastra appendiculata (D. Don) Makino & Bulb & low & Qiu et al. 2006 \\
\hline \multicolumn{4}{|l|}{ Papaveraceae } \\
\hline Chelidonium majus L. & Whole plant & medium & Zhu et al. 2006 \\
\hline Eomecon chionantha Hance & Whole plant & low & Qiu et al. 2006 \\
\hline Macleaya cordata (Willd.) R. Br. & Whole plant & high & Qiu et al. 2006 \\
\hline \multicolumn{4}{|l|}{ Phytolaccaceae } \\
\hline Phytolacca acinosa Roxb. & Root & medium & Zhu et al. 2006 \\
\hline \multicolumn{4}{|l|}{ Plumbaginaceae } \\
\hline Plumbago indica L. & Whole plant & medium & Anonymous, 1987 \\
\hline Plumbago zeylanica L. & $\begin{array}{l}\text { Root, leaf, } \\
\text { whole plant }\end{array}$ & medium & $\begin{array}{l}\text { Guo et al. 1990, Lin et al. } \\
\text { 2003, Qiu et al. } 2006\end{array}$ \\
\hline \multicolumn{4}{|l|}{ Polygonaceae } \\
\hline Polygonum hydropiper L. & Whole plant & medium & Zhu et al. 2006 \\
\hline \multicolumn{4}{|l|}{ Ranunculaceae } \\
\hline Aconitum carmichaeli Debeaux & Root tuber & high & Qiu et al. 2006, Zhu et al. 2006 \\
\hline Aconitum sinomontanum Nakai & Root & medium & Qiu et al. 2006 \\
\hline Anemone hupehensis (Lemoine) Lemoine & Whole plant, root & medium & Zhu et al. 2006 \\
\hline Anemone rivularis Buch.-Ham. ex DC. & Whole plant, root & low & $\begin{array}{l}\text { Anonymous, 1987, Guo et } \\
\text { al. 1990, Quu et al. } 2006\end{array}$ \\
\hline Ranunculus grandis Honda & Whole plant & medium & Zhu et al. 2006 \\
\hline \multicolumn{4}{|l|}{ Rhamnaceae } \\
\hline $\begin{array}{l}\text { Gouania leptostachya DC. } \\
\end{array}$ & Stem, leaf, root & medium & Lin et al. 2003 \\
\hline \multicolumn{4}{|l|}{ Rosaceae } \\
\hline Duchesnea indica (Andrews) Focke & Whole plant & Iow & Qiu et al. 2006, Zhu et al. 2006 \\
\hline Spiraea japonica L.f. & Root & low & Qiu et al. 2006 \\
\hline \multicolumn{4}{|l|}{ Rutaceae } \\
\hline Toddalia asiatica (L.) Lam. & Root, bark & low & $\begin{array}{l}\text { Lin et al. 2003, Qiu et al. } \\
\text { 2006, Zhu et al. } 2006\end{array}$ \\
\hline Zanthoxylum nitidum (Roxb.) DC. & Root, stem, leaf & low & Lin et al. 2003 \\
\hline Zanthoxylum planispinum Siebold \& Zucc. & Root, fruit & low & Quu et al. 2006 \\
\hline \multicolumn{4}{|l|}{ Saxifragaceae } \\
\hline Saxifraga stolonifera Curtis & Whole plant & low & Qiu et al. 2006, Zhu et al. 2006 \\
\hline \multicolumn{4}{|l|}{ Solanaceae } \\
\hline Datura stramonium L. & Root, leaf, fruit & low & Lin et al. 2003 \\
\hline Nicotiana tabacum L. & Leaf & medium & Guo et al. 1991, Qiu et al. 2006 \\
\hline Solanum americanum Mill. & Whole plant & medium & Lin et al. 2003, Qiu et al. 2006 \\
\hline Solanum hazenii Britton & Root, leaf, fruit & low & Lin et al. 2003 \\
\hline Solanum torvum Sw. & Root & Iow & Guo et al. 1991 \\
\hline
\end{tabular}




\section{Huai et al. - Ethnomedicinal Analysis of Toxic Plants from Five Ethnic Groups in China}

\begin{tabular}{|c|c|c|c|}
\hline Plant Family/Species & Part Used & Toxicity & Reference \\
\hline \multicolumn{4}{|l|}{ Stenonaceae } \\
\hline Stemona tuberosa Lour. & Root tuber & low & Guo et al. 1990 \\
\hline \multicolumn{4}{|l|}{ Theaceae } \\
\hline Camellia oleifera Abel & Seed & low & Qiu et al. 2006 \\
\hline \multicolumn{4}{|l|}{ Urticaceae } \\
\hline Urtica fissa E. Pritz. & Whole plant & low & Zhu et al. 2006 \\
\hline \multicolumn{4}{|l|}{ Valerianaceae } \\
\hline Valeriana jatamansi Jones & Root, rhizome & low & Zhu et al. 2006 \\
\hline \multicolumn{4}{|l|}{ Verbenaceae } \\
\hline Rotheca serrata (L.) Steane \& Mabb. & Whole plant & low & Lin et al. 2003 \\
\hline \multicolumn{4}{|l|}{ Vitaceae } \\
\hline Ampelopsis japonica (Thunb.) Makino & Root tuber & low & Zhu et al. 2006 \\
\hline
\end{tabular}

Table 2. Plant families with at least two toxic species out of 118 reported from Dai, Lahu, Miao, Tujia, and Wa ethnic groups in south and southwest China. Number of genera and species in each family and percent of the total genera and species reported. Overall diversity ranked by combined specific and generic diversity.

\begin{tabular}{|c|c|c|c|}
\hline Rank & Families & Genera & Species \\
\hline 1 & Araceae & $9(10.0 \%)$ & $13(11.0 \%)$ \\
\hline 2 & Asteraceae & $7(7.7 \%)$ & $8(6.8 \%)$ \\
\hline 3 & Fabaceae & $6(6.6 \%)$ & $7(5.9 \%)$ \\
\hline 4 & Euphorbiaceae & $5(5.5 \%)$ & $7(5.9 \%)$ \\
\hline 5 & Aristolochiaceae & $3(3.3 \%)$ & $6(5.1 \%)$ \\
\hline 6 & Apocynaceae & $5(5.5 \%)$ & $5(4.2 \%)$ \\
\hline \multirow[t]{2}{*}{7} & Ranunculaceae & $3(3.3 \%)$ & $5(4.2 \%)$ \\
\hline & Solanaceae & $3(3.3 \%)$ & $5(4.2 \%)$ \\
\hline 8 & Berberidaceae & $2(2.2 \%)$ & $5(4.2 \%)$ \\
\hline 9 & Papaveraceae & $3(3.3 \%)$ & $3(2.5 \%)$ \\
\hline 10 & Rutaceae & $2(2.2 \%)$ & $3(2.5 \%)$ \\
\hline \multirow[t]{2}{*}{11} & Chloranthaceae & $1(1.1 \%)$ & $3(2.5 \%)$ \\
\hline & Dioscoreaceae & $1(1.1 \%)$ & $3(2.5 \%)$ \\
\hline \multirow[t]{4}{*}{12} & Lauraceae & $2(2.2 \%)$ & $2(1.7 \%)$ \\
\hline & Liliaceae & $2(2.2 \%)$ & $2(1.7 \%)$ \\
\hline & Melanthiaceae & $2(2.2 \%)$ & $2(1.7 \%)$ \\
\hline & Rosaceae & $2(2.2 \%)$ & $2(1.7 \%)$ \\
\hline \multirow[t]{4}{*}{13} & Campanulaceae & $1(1.1 \%)$ & $2(1.7 \%)$ \\
\hline & Menispermaceae & $1(1.1 \%)$ & $2(1.7 \%)$ \\
\hline & Plumbaginaceae & $1(1.1 \%)$ & $2(1.7 \%)$ \\
\hline & Total & $62(68.1 \%)$ & $89(75.4)$ \\
\hline
\end{tabular}

At the generic level, $19.6 \%$ of toxic genera had more than one species. Genera with more than one species are listed in Table 3.
Table 3. Families and genera of toxic plants with multiple species reported as used medicinally by Dai, Lahu, Miao, Tujia, and Wa ethnic groups in south and southwest China.

\begin{tabular}{|l|l|c|}
\hline Families & Genera & Species \\
\hline \multirow{2}{*}{ Araceae } & Arisaema & 4 \\
\cline { 2 - 3 } & Typhonium & 2 \\
\hline Berberidaceae & Dysosma & 4 \\
\hline \multirow{2}{*}{ Aristolochiaceae } & Aristolochia & 3 \\
\cline { 2 - 3 } & Asarum & 2 \\
\hline Chloranthaceae & Croton & 2 \\
\cline { 2 - 3 } & Euphorbia & 2 \\
\hline Dioscoreaceae & Chloranthus & 3 \\
\hline Solanaceae & Dioscorea & 3 \\
\hline Asteraceae & Solanum & 3 \\
\hline Campanulaceae & Spilanthes & 2 \\
\hline Fabaceae & Lobelia & 2 \\
\hline Menispermaceae & Ormosia & 2 \\
\hline Plumbaginaceae & Stephania & 2 \\
\hline Ranunculaceae & Plumbago & 2 \\
\hline Rutaceae & Aconitum & 2 \\
\cline { 2 - 3 } & Anemone & 2 \\
\hline & Zanthoxylum & 2 \\
\hline
\end{tabular}

The parts of toxic plants used as ethnomedicines included roots, whole plant and leaf, flowers, fruit, and seeds. Roots were most frequently used. In some cases, parts of toxic plants used as medicines were not the main parts containing toxins. For example, the Dai people used the bark and leaf of Antiaris toxicaria Lesch. as medicine, while its gum contained deadly toxins. As another example, the leafs of Plumbago indica L. were considered to be very toxic by 
Lahu people, its whole plant, however, was often used as medicines.

Family is an important taxonomic classification level for determining the usefulness of plant species to local people (Thomas et al. 2009). This is also true for the toxicity of plants. In the 5 ethnomedicine systems, many toxic plants belong to just a few families (Araceae, Asteraceae, Fabaceae, Euphorbiaceae, Aristolochiaceae, and Apocynaceae). These are among the main families containing toxic plants (Levetin \& McMahon 2008). Although some families, such as Lobeliaceae, Loganiaceae and Moraceae, have only a few species with toxicity, the toxins from them are deadly poisonous. Huai et al. (2003) reported that Lobelia clavata E. Wimm. and Gelsemium elegans (Gardner \& Champ.) Benth. were considered as two of the most poisonous plants in the Autonomous County by Jinping Miao, Yao and Dai in Yunnan Province in China. They were also used medicinally by the Lahu, Hani, Yao people (Huai et al. 2003).

\section{Toxic plants used the most frequently in the five ethnic groups}

In spite of living in the same environments, different ethnic groups have their own knowledge and experiences of using toxic plants as medicines. We compared the plants used by the five groups, and found that some toxic plants were used as medicines by two or more ethnic groups, and some were used only by single ethnic group. For instance, castor (Ricinus communis L.) was used by four ethnic groups (Wa, Dai, Tujia and Miao) (Table 4). Ricinus communis is cultivated commonly in south and southwest China. This fact might be the main reason why it was used frequently. Plumbago zeylanica L., Anemone rivularis Buch.-Ham. ex DC., Toddalia asiatica (L.) Lam., and Paris polyphylla Sm. were used in three of the five ethnomedicinal systems (Table 4).

UVs and AUVs showed that toxic plants used as medicines by the five ethnic groups had high medicinal use values (Table 5). This result indicated that toxic plants used as medicines were an important content of the five ethnomedicinal systems. Each toxic plant used had at least two different medicinal usages (Table 4). Of them, Anemone rivularis had the highest UVs value, and Croton tiglium $L$. had the lowest UVs value. This indicates that $A$. rivularis had the most multiple usages in different ethnomedicinal systems. Although $C$. tiglium was used by two ethnic groups, it was used only for two health problems. The UVs of toxic plants were not correlated closely with the number of ethnic groups who used them. The use number of a plant contributed greatly to the UVs. Compared with UVs, AUVs were better at reflecting the relative use values of toxic plants in ethnomedicine. For example, the plants $R$. communis, Alangium chinense (Lour.) Harms, Coriaria sinica Maxim., Dioscorea hispida Dennst., Euphorbia lathyria L., and Curculigo orchioides Gaertn. shared the same
Table 4. The most frequent cited toxic plants used by Dai, Lahu, Miao, Tujia, and Wa ethnic groups in south and southwest China. UV (Use Value); AUV (Average Use Value)

\begin{tabular}{|c|c|c|c|}
\hline Species & $\begin{array}{l}\text { Number of } \\
\text { ethnic groups } \\
\text { using plant (\%) }\end{array}$ & UV & $A U V$ \\
\hline Ricinus communis & $4(80 \%)$ & 4.00 & 3.20 \\
\hline Anemone rivularis & \multirow{4}{*}{$3(60 \%)$} & 9.00 & 5.40 \\
\hline Paris polyphylla & & \multirow{2}{*}{6.33} & \multirow{2}{*}{3.79} \\
\hline Plumbago zeylanica & & & \\
\hline Toddalia asiatica & & 5.00 & 3.00 \\
\hline Duchesnea indica & \multirow{24}{*}{$2(40 \%)$} & 7.50 & 3.00 \\
\hline Aconitum carmichaeli & & 6.50 & 2.60 \\
\hline Arisaema erubescens & & 6.00 & 2.40 \\
\hline Melia azedarach & & \multirow{5}{*}{5.50} & \multirow{5}{*}{2.20} \\
\hline Nicotiana tabacum & & & \\
\hline Rohdea japonica & & & \\
\hline Saxifraga stolonifera & & & \\
\hline Spilanthes callimorpha & & & \\
\hline Cassytha filiformis & & \multirow{9}{*}{4.00} & \multirow{4}{*}{1.80} \\
\hline Dysosma veitchii & & & \\
\hline Euphorbia antiquorum & & & \\
\hline Gelsemium elegans & & & \\
\hline Alangium chinense & & & \multirow{5}{*}{1.60} \\
\hline Coriaria sinica & & & \\
\hline Curculigo orchioides & & & \\
\hline Dioscorea hispida & & & \\
\hline Euphorbia lathyria & & & \\
\hline Solanum nigrum & & 3.50 & 1.40 \\
\hline Dioscorea bulbifera & & \multirow{4}{*}{3.00} & \multirow{4}{*}{1.20} \\
\hline Pachyrhizus erosus & & & \\
\hline Pinellia ternat & & & \\
\hline Remusatia vivipara & & & \\
\hline Millettia pachycarpa & & 2.50 & 1.00 \\
\hline Croton tiglium & & 2.00 & 0.80 \\
\hline
\end{tabular}

UVs, 4.00, but had different AUVs. The AUV of R. communis was higher than that of the other species. Obviously, some toxic plants were used only by a single ethnic group, and others were commonly used by two or more ethnic groups. This meant that different ethnic groups had their own ethnobotanical knowledge reflected by their different choices and preferred using different toxic plants. 


\section{Huai et al. - Ethnomedicinal Analysis of Toxic Plants from Five Ethnic Groups in China}

Table 5. Plants with the "highest" toxicity used medicinally by Dai, Lahu, Miao, Tujia, and Wa ethnic groups in south and southwest China and their Use Values (UV) and Averge Use Values (AUV).

\begin{tabular}{|l|l|l|l|}
\hline Species & $\begin{array}{l}\text { Ethnic groups } \\
\text { using plants }\end{array}$ & UV & AUV \\
\hline Aconitum carmichaeli & Tujia, Miao & 6.50 & 2.60 \\
\hline Gelsemium elegan & Wa, Lahu & 4.50 & 1.80 \\
\hline Remusatia vivipara & Wa, Lahu & 3.00 & 1.20 \\
\hline Macleaya cordata & Miao & 6.00 & 1.20 \\
\hline Millettia pachycarpa & Wa, Lahu & 2.50 & 1.00 \\
\hline $\begin{array}{l}\text { Arisaema } \\
\text { consanguineum }\end{array}$ & Tujia & 4.00 & 0.80 \\
\hline Croton tiglium & Tujia, Dai & 2.00 & 0.80 \\
\hline Gloriosa superba & Dai & 3.00 & 0.60 \\
\hline Antiaris toxicaria & Dai & 3.00 & 0.60 \\
\hline Nerium indicum & Tujia & 3.00 & 0.60 \\
\hline Lobelia clavata & Wa & 3.00 & 0.60 \\
\hline
\end{tabular}

\section{The high toxic plants and their usages among ethnic groups}

Eleven species were believed to be extraordinarily toxic by the five ethnic groups (Table 5). Due to their extreme toxicity, these toxic plants often were used only by local species (Table 5 ), were only shared as medicines by two ethnic groups. The Miao specialist used only two high toxic plants Aconitum carmichaeli Debeaux and Macleaya cordata (Willd.) R. Br. while Gloriosa superb L. and A. toxicaria were used as medicines only by Dai healers. Previously, Huai et al. (2003) reported L. clavata and G. elegans as being highly toxic plants used in the Miao, Yao and Dai society in Yunnan of China. This survey confirmed their medicinal use in these ethnic groups.

These 11 plants also had high UVs. They were all above or equal to 2.00 with the UV and AUV of $A$. carmicharli being the highest. Although M. cordata had a high UV, 6.00, specialists to treat special health problems. Five of these

Table 6. The top 15 indications treated with toxic plants and the number of concerned plants used by Dai, Lahu, Miao, Tujia, and Wa ethnic groups in south and southwest China.

\begin{tabular}{|l|c|}
\hline Usage & Species (\%) \\
\hline Injuries from falls* & $94(78.3)$ \\
\hline Skin problems** & $84(70.0)$ \\
\hline Snakebite & $34(28.3)$ \\
\hline Stomach ache & $34(28.3)$ \\
\hline Rheumatoid arthritis & $32(26.7)$ \\
\hline Tussis & $21(17.5)$ \\
\hline Burn and scald & $19(15.8)$ \\
\hline Throat inflammation & $16(13.3)$ \\
\hline Lymph inflammation & $10(8.3)$ \\
\hline Malaria & $9(7.5)$ \\
\hline Parotitis & $8(6.7)$ \\
\hline Menoxenia & $7(5.8)$ \\
\hline Cold & $7(5.8)$ \\
\hline Anesthesia & $7(5.8)$ \\
\hline Diarrhea & $7(5.8)$ \\
\hline
\end{tabular}

* including injuries from falls, bone broken, and bleeding caused by trauma.

** including various ailments related to skin.

its AUV was only 1.20 , lower than G. elegan. Croton tiglium had the lowest UV in these 11 plants, 2.00, but its AUV was not the lowest. These results indicated that the plants with "highest" toxicity also played a very important role in some ethnomedicinal systems, although many of them were not commonly used by all ethnic groups concerned.

The use of highly toxic plants may mean some special beneficial toxin exists in these plants. The high AUVs and UVs indicated that these medicinal plants might have a potential to develop new drugs. Thus these highly toxic plants used as medicines are particularly interesting in further research or new drug development.

Figure 1. Hierachical cluster dendrogrm of Dai, Lahu, Miao, Tujia, and Wa ethnic groups in south and southwest China. based on their use of toxic plants. Produced using SPSS 16.0 applying a Pearson's product-moment correlation (SPSS 2008).

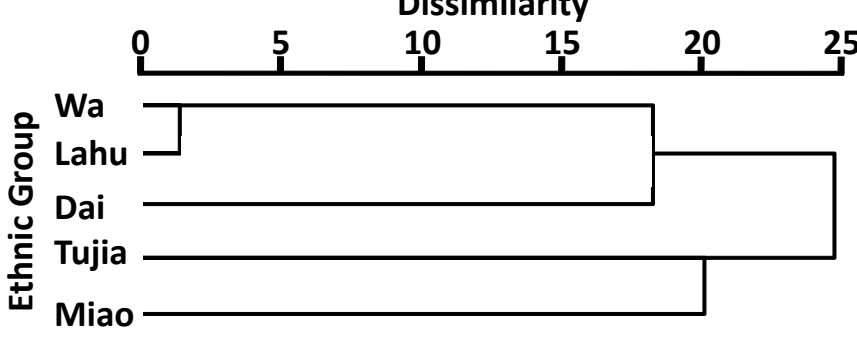

\section{Health problems and treatment with toxic plants}

Toxic plants often were used for different medical purposes among different ethnic groups. Some health problems were treated preferentially with toxic plants across the five ethnic groups. The frequent health problems treated with toxic plants in these ethnic groups and the numbers of toxic plants involved are shown in Table 6. Injuries (including bleeding caused by trauma and broken bones) were the most frequent problems treated, with 94 toxic plants $(78.33 \%$ of total toxic plants) from the five ethnic groups. Skin problems were the second most frequent illness treated 
with 84 toxic plants $(70 \%)$. The toxic plants used for injuries and dermatological medicines were significantly higher than for other health problems $(u=6.465, p<0.01)$. Although treatment of skin ailments frequently involved toxic plants in the five ethnic groups, these indigenous people had different experiences and uses for toxic plants for treatment of skin ailments. It is usually difficult to define "what is a skin ailment?" and "which skin problems were included within skin ailments?". When the medicinal specialists from different ethnic groups answer these questions, the answers usually differed from one another. Here, the skin problems defined included various ailments related to skin problems in local areas. In addition, some toxic plants were used for treating snakebite, stomachache, rheumatoid arthritis, tussis, burns, scalds, malaria, colds, throat inflammation, and diarrhea. In another word, the use of toxic plants was usually correlated closely with specific health problems in local communities.

It is common that the same toxic plants were used by different ethnic groups to treat the same health problems. Since a given ethnomedicine system was usually developed and practiced independently with unique cultural backgrounds for ages, the context of the given ethnomedicine formed its own specializations, though their practitioners might live in the same areas. The same species was often used by different ethnic healers to treat the same health problems. This was likely to be a result of experience exchange due to its high medicinal effectiveness. Thus, plants used more often by different ethnic healers to treat the same ailment should be the focus of more attention during the hunt for new drugs.

Toxicity of toxic plants depends on their chemical composition. Alkaloids are one of the main components that can cause poisoning. For example, more than 40 alkaloids were isolated from G. elegans (Hua et al. 2008), which could cause violent clonic convulsions leading to respiratory failure (Rujjianawate et al. 2003); benzofuran lignan glycosides isolated from $G$. elegans showed a potent cytotoxic activity (Hua et al. 2008); The diterpenoid alkaloids were thought to be the main toxin in $A$. carmichaeli (Konno et al. 1982); dioscorine and sapogenins isolated from Aconitum also showed cytotoxic activity (Webster et al. 1984); plumbagin isolated from $P$. zeylanica, presented significant cytoxicity (Lin et al. 2003). Medicinal activity of toxic plants also depended on its composition. Benzophenanthridine alkaloids, coumarins, cyclohexylamides and terpenoids isolated from $T$. asiatica showed various medicinal activities, including strong anti-platelet aggregation activity (Tsai et al. 1998), antibacterial and antifungal activities (Duraipandiyan \& Ignacimuthu 2009), and anticancer (Rajkumar et al. 2008).

Crude extracts with uncertain composition have also been shown to be biologically active from many toxic plants. Duchesnea indica (Andrews) Focke extract showed strong anti-inflammatory activity, in particularly suppress- ing pro-inflammatory cytokines and mediator by blocking NF-kB activation (Zhao et al. 2008). The crude extracts from $P$. zeylanica could increase the activities of serum lactate dehydrogenase, acid phosphatase, liver alkaline phosphatase, and sugar concentrations in the blood in clinic experiments (Beg \& Ahamd 2000). Identifying the compounds responsible for the activities in these toxic plants is the premise of better understanding and using toxic plants.

\section{Conclusion}

Based on this study, the culture and the resources available were two main factors affecting ethnic healers' selection choices for uses of toxic plants. Although the active composition of toxic plants used medicinally by the five ethnic groups was uncertain, our current survey may provide interesting clues to further understand and apply these toxic plants.

\section{Acknowledgements}

This research was supported by the National Natural Science Foundation of China (Grant No. 30770220). The authors are grateful to two anonymous reviewers for their valuable comments on this paper.

\section{Literature Cited}

Al-Qura'n, S. 2005. Ethnobotanical survey of folk toxic plants in southern part of Jordan. Toxicon 46:119-129.

Arias, A.B.T. 2000. Ichthyotoxic plants used in Spain. Journal of Ethnopharmacology 73:505-512.

Beg, A.Z. \& I. Ahmad. 2000. Effect of Plumbago zeylanica extract and certain curing agents on multidrug resistant bacteria of clinical origin. World Journal of Microbiology \& Biotechnology 16:841-844.

Bhattarai, S., Chaudhary, R.P. \& R.S.L. Taylor. 2009. Wild edible plants used by the people of Manang district, Central Nepal. Ecology of Food and Nutrition 48(1):1-20.

Duraipandiyan, V. \& S. Ignacimuthu. 2009. Antibacterial and antifungal activity of Flindersine isolated from the traditional medicinal plant, Toddalia asiatica (L.) Lam. Journal of Ethnopharmacology 123:494-498.

Eddouks, M., M. Maghrani, A. Lemhadri, M.L. Quahidi \& H. Joud. 2002. Ethnopharmacological survey of medicinal plants used for the treatment of diabetes mellitus, hypertension and cardiac diseases in the south-eastern region of Morocco (Tafilalet). Journal of Ethnopharmacology 82:97-103. 


\section{Huai et al. - Ethnomedicinal Analysis of Toxic Plants from Five Ethnic Groups in China}

Farnsworth, N.R. 1966. Biological and phytochemical screening of plants. Journal of Pharmaceutical Sciences 55:225-276.

Guo, S., H. Duan \& D. Guo. 1990. Si Ndah Ba Raog Joung Gox (The Ethnomedicines of the Wa in China) (I). Yunnan Nationality Press, Kunming.

Guo, D., S. Guo \& H. Duan. 1991. Si Ndah Ba Raog Joung Gox (The Ethnomedicines of the Wa in China) (II). Yunnan Nationality Press, Kunming.

Habermehl, G.G. 2004. Highlights on plant toxins in Toxicon. Toxicon 44(4):341-344.

Harvey, A.L., K.N. Bradley, S.A. Cochran, E.G. Rowan, J.A. Quillfeldt \& D.A Jerusalinsky. 1998. What can toxins tell us for drug discovery. Toxicon 36:1635-1640.

Heinrich, M. 2000. Plant resources of south-east Asia medicinal and poisonous plants 1. Phytochemistry 53:619620.

Hua, W., Q.C. Zhao, J. Yang, G.B. Shi, L.J. Wu \& T. Guo. 2008. Two new benzofuran lignin glycosides from Gelsemium elegans. Chinese Chemical Letters 19:13271329.

Huai, H. \& J. Xu. 2000. Indigenous knowledge: an inexhaustible "information bank" for toxin research. Toxicon 38(6):745-746.

Huai, H., S. Yang \& S. Pei. 2003. Ethnobotany of two "toxic" plants: Lobelia clavata and Gelsemium elegans in Southeast Yunnan, China. Ethnobotany 15:20-22.

Konno, C., M. Shirasaka \& H. Hikino. 1982. Structure of senbusine $\mathrm{A}, \mathrm{B}$ and $\mathrm{C}$, diterpenic alkaloids of Aconimtu carmichaeli roots from China. Journal of Natural Products 45(2):128-133.

Levetin, E. \& K. McMahon. 2008. Plants \& Society. 5th edition. McGraw-Hill, New York.

Lin L.C., L.L. Yang \& C.J. Chou. 2003. Cytotoxicity naphthoquinones and plumbagic acid glucosides from Plumbago zeylanica. Phytochemistry 62(4):619-622.

Lin, Y., Z. Yi \& Y. Zhao. 2003. Chinese Dai Medicine Colorful Illustrations. Yunnan Nationality Press, Kunming.

Neuwinger, H.D. 2004. Plants used for poison fishing in tropical Africa. Toxicon 44(4):417-430.

Phillips, O. \& A.H. Gentry. 1993a. The useful plants of Tambopata, Peru: I. Statistical hypotheses tests with a new quantitative techniques. Economic Botany 47:15-32.
Phillips, O. \& A.H. Gentry. 1993b. The useful plants of Tambopata, Peru: II. Additional hypotheses testing in quantitative ethobotany. Economic Botany 47:33-43.

Qiu, D., J. Du \& T. Xia. 2006. Zhonghua Bencao Miaoyaojuan Caise Tupu (Chinese Herbals-Colorful Illustrations of the Miao). Publishing House of Ancient Chinese Medical Books, Beijing.

Rajkumar, M., R.H. Chandra, K. Asres \& C. Veeresham. 2008. Toddalia asiatica (Linn.) Lam. - a comprehensive review. Pharmacognosy Reviews 2(4):386-397.

Rates, S., 2001. Plants as source of drugs. Toxicon 39 (5):603-613.

Rios, J.L. \& P.G. Waterman. 1998. A review of the pharmacology and toxicology of Astragalus. Phytotherapy Research 11:411-418.

Rujjianawate, C., D. Kanjanapothi \& A. Panthong. 2003. Pharmacological effect and toxicity of alkaloids from Gelsemium elegans Benth. Journal of Ethnopharmacology 89:91-95.

Simao Institute of Ethnomedicine. 1987. The Commonly Used Medicine of the Lahu. Yunnan Nationality Press, Kunming.

SPSS. 2008. Statistical Package for Social Sciences. SPSS for Windows, Rel. SPSS 16.0.2. 2008. Chicago: SPSS Inc.

Stinson, S. 1992. Nutritional Adaptation. Annual Review of Anthropology 21:143-170.

Thomas E., I. Vandebroek, S. Sanca \& P.V. Damme. 2009. Cultural significance of medicinal plant families and species among Quechua framers in Apillampa, Bolivia. Journal of Ethnophamacology 122:60-67.

Tsai, I.L., M.F. Wun, C.M. Teng, T. Ishikawa \& I.S. Chen. 1998. Anti-platelet aggregation constituents from formosan Toddalia asiatica. Phytochemistry 48(8):1377-1382.

Vetter, J. 2000. Plant cyanogenic glycosides. Toxicon 38:11-36.

Wang, J., F. He, C. Hzang, W. Fong, P. Xiao, R. Han \& M. Yang. 2005. Differential gene expression profiles in paclitaxel-induced cell cycle arrest and apoptosis in human breast cancer MCF-7 cells. Acta Pharmaceutica Sinica 40 (12):1099-1104.

Webster, J., W. Beck \& B. Ternai. 1984. Toxicity and bitterness in Australian Dioscorea bulbifera L. and Dioscorea hispida Dennst. from Thailand. Journal of Agricultural and Food Chemistry 32(5):1087-1090. 
Wilson, C.R., J.M. Sauer \& S.B. Hooser. 2001. Taxines: A review of the mechanism and toxicity of yew (Taxus spp.) alkaloids. Toxicon 39 (2-3):175-185.

Zhang, S. 1996. Cokawr Ladhofnatzhid Lir (The Ethnomedicines of the Lahu in China). Yunnan Nationality Press, Kunming.
Zhao, L., S.L. Zhang, J.Y. Tao, F. Jin, R. Pang, Y.J. Guo, P. Ye, J.H. Dong \& G.H. Zheng. 2008. Anti-imflammatory mechanism of a folk herbal medicine, Duchesnea indica (Andr.) Focke at RAW264.7 cell line. Immunological Investigations 37:339-357.

Zhu, G., J. Du \& J. Zhang. 2006. The Ethnomedicines of the Tujia. Publishing House of Ancient Chinese Medical Books, Beijing. 